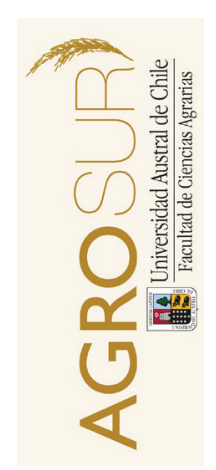

\title{
Propiedades físicas e hidráulicas de suelo y su relación con el movimiento de contaminantes
}

\author{
Physical and hydraulic properties of soil and their relationship \\ with the movement of pollutants
}

\author{
Seguel, $0 .^{a^{*}}$, Fuentes, I. ${ }^{a}$, Dörner, $\mathrm{J}^{b}$, Cuevas, J. ${ }^{c}$ \\ ${ }^{a}$ Universidad de Chile, Facultad de Ciencias Agronómicas, Departamento de Ingeniería y Suelos. \\ ${ }^{\mathrm{b}}$ Universidad Austral de Chile, Facultad de Ciencias Agrarias, Instituto de Ingeniería Agraria y Suelos. \\ Centro de Investigación en Suelos Volcánicos (CISVo) \\ ${ }^{\text {c} U n i v e r s i d a d ~ d e ~ C o n c e p c i o ́ n, ~ F a c u l t a d ~ d e ~ A g r o n o m i ́ a, ~ D e p a r t a m e n t o ~ d e ~ S u e l o s ~ y ~ R e c u r s o s ~ N a t u r a l e s . ~}$
}

\begin{abstract}
A B S T R A C T
This review considers the physical principles of soil related to movement of pollutants. Considering that most of the potential contaminants are water soluble or can be displaced by water flux, clearly the movement of these agents through the soil is associated to water movement. Therefore it is very important to know the soil characteristics that determine the organization of the porous system (texture, structure, organic matter content) which ultimately determine its diameter, continuity and connectivity to the microcosm of pollution. It is important to consider other soil properties, such as compaction and hydrophobicity that influence heterogeneity of the media and preferential flow zones. The work scale, the spatial variability and the nature of pollutant determine the strategies of study, prevention and control of the contaminants; this review shows a brief review of a laboratory method and reaction processes of pollutants in soil. Gas flows could not be left out of the analysis, especially with regard to the emission of greenhouse gases. Finally, the review leaves open to controversy the subject of the role of soil as an environmental filter.
\end{abstract}

\section{RESUMEN}

El presente trabajo presenta una revisión de los principios de física de suelo relacionados con el movimiento de agentes con potencial contaminante. Considerando que gran parte de los contaminantes son solubles en agua o pueden ser desplazados con el flujo de ésta, el movimiento de estos agentes en el suelo está asociado al flujo de agua, por lo que resulta de gran importancia conocer las características del suelo que determinan la organización del sistema poroso (textura, estructura, contenido de materia orgánica) y que en definitiva determinan su diámetro, continuidad y conectividad hacia el microcosmos de contaminación. Es importante tener presente otras características del suelo, como la compactación y la hidrofobicidad, que influyen en la heterogeneidad del medio y en las zonas de flujo preferencial. La escala de trabajo, la variabilidad espacial y la naturaleza del contaminante determinan las estrategias de estudio, de prevención y de control de contaminantes, haciéndose una revisión somera de un método de laboratorio y de los procesos de reacción de los contaminantes con el suelo. Igualmente no se pueden dejar de lado los flujos gaseosos, sobre todo en lo que respecta a la emisión de gases de efecto invernadero. Finalmente el trabajo deja abierta la polémica con respecto al rol del suelo como filtro ambiental.

Palabras clave: Movimiento de agua en el suelo, contaminación difusa, curvas de penetración.

\section{INTRODUCCIÓN}

Existe un sinnúmero de compuestos potencialmente peligrosos en el ambiente, que al ser depositados en el suelo podrían afectar su calidad o, en el peor de los casos, contaminar acuíferos o el aire en forma no controlada. No solo las actividades industriales son fuentes de contaminación, la agricultura, a través de procesos de contaminación difusa, puede aportar grandes can- tidades de contaminantes al medio, como por ejemplo pesticidas, fertilizantes, purines, etc. (Bohne, 2005). Sería preferible que algunos contaminantes, como los metales pesados, permaneciesen estables en el lugar de origen, pero ya sea por acidificación, actividad biológica, erosión, etc., pueden disolverse y/o movilizarse. El transporte de solutos en medios porosos, si bien se puede abordar desde diversos enfoques, posee principios básicos comunes que se explican a través de la 
física de suelos. La movilización tiene un significado amplio y describe una amplia gama de procesos (Haygarth y Jarvis, 1999; Trudgill et al., 1991), por lo que se hace necesario dividirla en dos componentes: solubilización (liberación de sustancias por procesos químicobiológicos como la mineralización) y separación (que involucra procesos físico-mecánicos como la erosión) en un modelo continuo fuente-movilización-transporte-impacto, que permite comprender el concepto de contaminación difusa (Haygarth et al., 2005). Considerando que gran parte de los contaminantes se mueven fácilmente a través de la fase líquida, el presente trabajo tiene por objetivo realizar una revisión de las bases del flujo de agua en el suelo que permitan comprender tanto el transporte de solutos como los procesos de interacción de los contaminantes con la fase sólida.

\section{Contaminantes en el suelo: flujo asociado al agua}

Considerando la ecuación de conservación para desarrollar un balance de masas que explique el movimiento macroscópico de agua en el suelo, es fácil adaptar dicho enfoque a un agente químico de interés, donde la expresión de la ecuación de conservación en una dimensión queda dada por:

$$
\partial \mathrm{C}_{\mathrm{T}} / \partial \mathrm{t}+\partial \mathrm{J}_{\mathrm{c}} / \partial \mathrm{z} \pm \mathrm{r}_{\mathrm{c}}=0
$$

Donde $\mathrm{C}_{\mathrm{T}}$ es la concentración total del químico en todas sus formas (masa del agente/volumen de suelo), $\mathrm{J}_{\mathrm{c}}$ es el flujo de masas (masa del agente/área - tiempo) $\mathrm{y}_{\mathrm{c}}$ es la tasa de cambio de masa por unidad de volumen como consecuencia de reacciones con el medio (Jury y Horton, 2004), y que puede ser considerado como una fuente $\left(r_{c}>0\right)$ o como un sumidero $\left(r_{c}<0\right)$ (Leij y van Genuchten, 2002). En caso que el agente químico esté también en fase gaseosa, su transporte será principalmente por difusión, por lo que se regirá por la ley de Fick (Jury y Horton, 2004; Maier y Schack-Kirchner, 2014), aunque en algunas circunstancias los procesos de convección pueden ser muy efectivos (Campbell, 1995).

La convección o flujo de masas denota el proceso por el cual un soluto es transportado por el flujo de agua. Para el caso de un capilar, el movimiento del agua está regido por la Ley de Poiseuille (Figura 1):

$$
\mathrm{Q}=\left(\pi \mathrm{R}^{4} \Delta \mathrm{P}\right) /(8 \mathrm{~L} \eta)
$$

Donde $Q$ es el flujo de agua, $\mathrm{R}$ es el radio del capilar, $\Delta \mathrm{P}$ es el diferencial de presión hidrostática a través de la longitud L del capilar y $\eta$ es la viscosidad del fluido. Resalta en esta expresión el hecho de que permite entender que un capilar de radio $2 \mathrm{R}$ tendrá 16 veces mayor flujo de agua por unidad de tiempo que un capilar de radio $\mathrm{R}$, denotando el riesgo de suelos con sistemas porosos gruesos como posibles agentes contaminantes (Pinochet, 2000).

Sin embargo, tal como lo expresa la Figura 1 (derecha), el suelo no es un sistema de capilares de radio constante, sino que presenta una gran variabilidad en forma, tamaño y continuidad del sistema poroso, por lo que la aproximación a los flujos de agua resulta más adecuada a través de una mirada macroscópica. La solución más conocida para entender el flujo de agua en el suelo fue desarrollada por Darcy (1856) para medios porosos en condición saturada (Figura 2).

La Ley de Darcy establece que el flujo de agua dentro de un sistema poroso depende en forma directa del diferencial de presión $(\Delta \mathrm{h})$ y en forma inversa del largo (l) de la columna de suelo; sin embargo, existe un factor de proporcionalidad (k) que explica el flujo de salida (q). Dicho factor es la conocida conductividad hidráulica $(\mathrm{K})$, y con análisis posteriores se demostró que depende tanto de la naturaleza del medio conductor (arreglo del sistema poroso) como de las propiedades del fluido (Jury et al., 1991). Sin embargo, la mayoría de los suelos agrícolas no se encuentran saturados, por lo que la capacidad de flujo del suelo se ve disminuida en la medida que existen menos poros conductores, disminuyendo la K a medida que el suelo se seca (Figura 3).

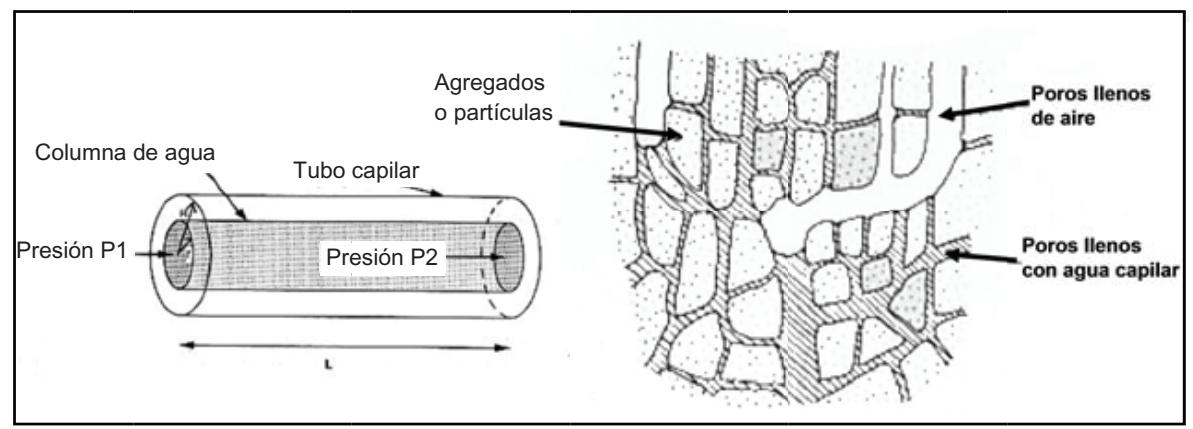

Figura 1. A la izquierda, el flujo de agua en un tubo capilar; a la derecha, representación esquemática de un sistema de poros y su tortuosidad.

Figure 1. On the left, water flux in a capillary tube; on the right, schematic representation of porous system and their tortuosity. 


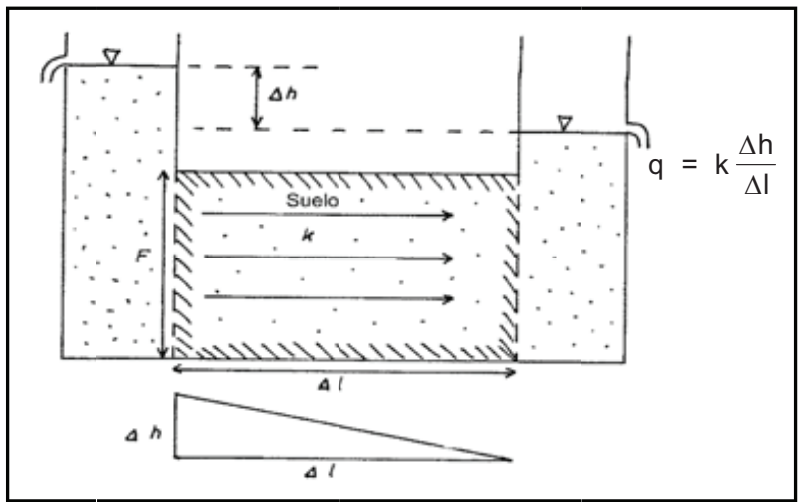

Figura 2. Columna de suelo para ilustrar la Ley de Darcy con el flujo (q) de salida de agua. Adaptado de Hartge y Horn (2009).

Figure 2. Soil column used to illustrate Darcy's law with the exit flux (q) of water. Adapted from Hartge and Horn (2009).

Como se observa en la Figura 3, la capacidad de flujo es máxima en un suelo saturado, lo que implica altos riesgos de contaminación en suelos con presencia de agua libre, en que la atracción de gravedad drena el agua en exceso a través de la porosidad gruesa (Hartge y Horn, 2009). Existe un efecto del tamaño de las partículas que componen la matriz del suelo (Figura 3, izquier$\mathrm{da}$ ), donde los materiales arenosos poseen una mayor $\mathrm{K}$ saturada, aumentando el riesgo de contaminación por percolación profunda. Sin embargo, al desaturarse, los suelos arenosos pierden continuidad y su K no saturada es menor en relación a suelos de texturas finas (Scheffer y Schachtschabel, 2002), en cuyo caso el agua es retenida a mayores tensiones en microporos formados por el arreglo de las partículas primarias y donde forma capas continuas que rodean a las partículas de arcilla.

Este comportamiento se repite en función de la estructura, donde suelos bien estructurados poseen una alta K saturada, en tanto suelos no estructurados o compactados presentan una disminución de la $\mathrm{K}$ de forma más gradual a medida que el suelo se seca (Hillel, 1980). La Figura 3 (derecha) presenta resultados prácticos de medición de K no saturada mediante infiltrómetros de disco, en el que se comprobó que la mejor estructuración del suelo franco limoso en relación al franco arenoso generó una alta $\mathrm{K}$ en condiciones cercanas a saturación, pero esta cayó rápidamente al secarse el suelo. En términos prácticos, la proyección de las rectas hasta el valor clásico de capacidad de campo (tensión equivalente a $330 \mathrm{~cm}$ ) permite corroborar que la $\mathrm{K}$ disminuye en varios órdenes de magnitud en relación a la K saturada, lo que ha sido comprobado para distintos tipos de suelos y bajo distintas condiciones de manejo (Nissen et al., 2006; Dörner et al., 2009; Keller, 2012; Cuevas et al., 2013). En definitiva, un suelo con un contenido de agua menor a capacidad de campo posee un bajo riesgo de movimiento de contaminantes por agua, dada la baja $\mathrm{K}$ en estas condiciones; sin embargo, en esa situación se generan las condiciones de aireación que favorecen procesos de volatilización o de transformación biológica (Stepniewska et al., 2004).

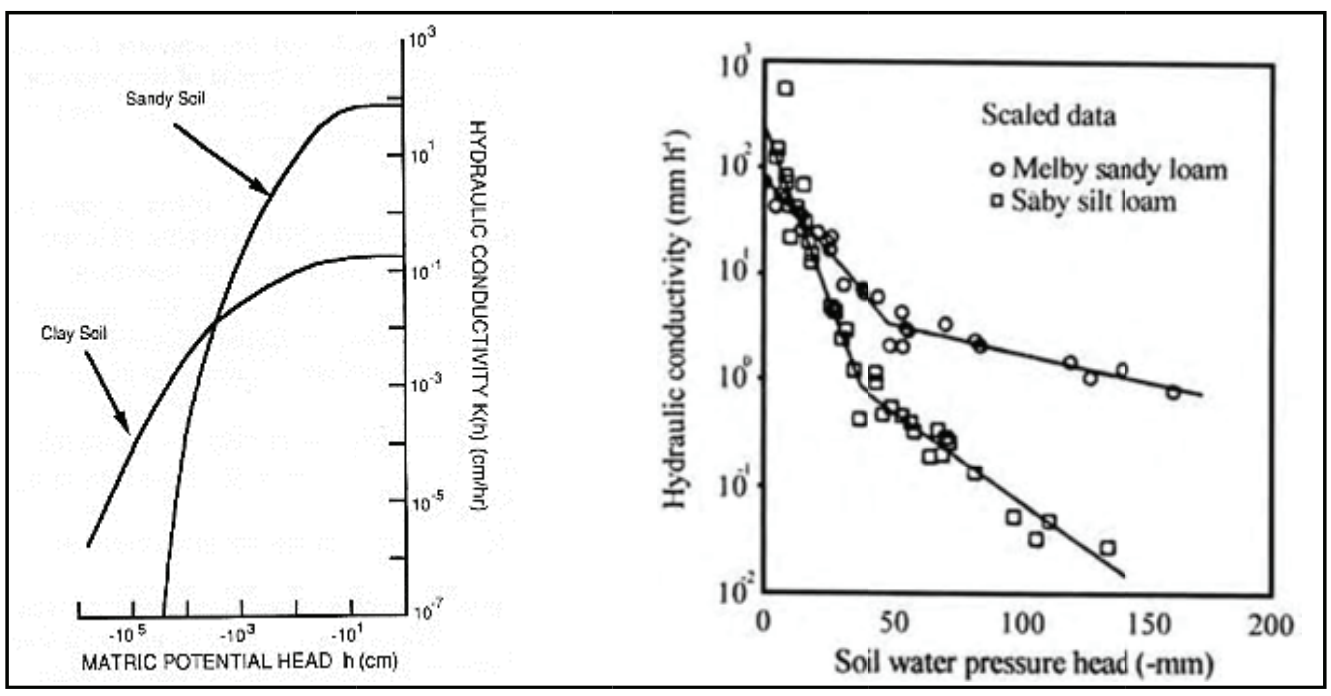

Figura 3. Variación de la conductividad hidráulica $(K)$ en función de la tensión mátrica del agua del suelo (izquierda). A la derecha, valores reales de K obtenidos en suelos franco arenoso (círculo) y franco limoso (cuadrado). Adaptado de Jury et al. (1991, izquierda) y de Jarvis y Messing (1995, derecha).

Figure 3. Variation of hydraulic conductivity $(\mathrm{K})$ in function of matric tension (left). At the right, real values of $\mathrm{K}$ obtained in sandy loam (circles) and silty loam (squares) soils. Adapted from Jury et al. (1991, left) and Jarvis and Messing (1995, right). 


\section{Procesos en el suelo durante el movimiento de contaminantes}

Los solutos en el suelo no se mueven sólo por flujos de masa, también existen otros mecanismos, algunos de los cuales se detallan en la Figura 4.

La dispersión hidrodinámica es el resultado del flujo de una solución no homogénea a través de un cuerpo poroso, el cual produce tanto mezclas como diferencias entre las distintas porciones de la solución (Hillel, 1980). Así, el flujo en capilares gruesos es mayor que en capilares finos, y en cada uno de ellos se genera una velocidad de flujo no uniforme a nivel microscópico; sin embargo, no solo resulta importante el diámetro de los poros, sino su continuidad y tortuosidad (Hillel, 1998). Este fenómeno explica que en los poros finos existe un retardo en la aparición del flujo de salida (Bohne, 2005). Algunos autores consideran que la dispersión hidrodinámica cuantifica los efectos de la dispersión y la difusión, especialmente cuando se mezclan porciones de soluciones distintas (Leij y van Genuchten, 2002), pero acá se considerarán como fenómenos por separado.

Debido a que el resultado en la distribución de la solución depende de las diferencias de concentración, el flujo causado por la dispersión hidrodinámica $\left(\mathrm{J}_{\mathrm{h}}\right)$ puede ser descrito por:

$$
J_{h}=-\theta D_{h} \cdot d c / d x
$$

Donde $\mathrm{D}_{\mathrm{h}}$ es el coeficiente de dispersión (en unidades de área / tiempo), $\theta$ es el contenido volumétrico

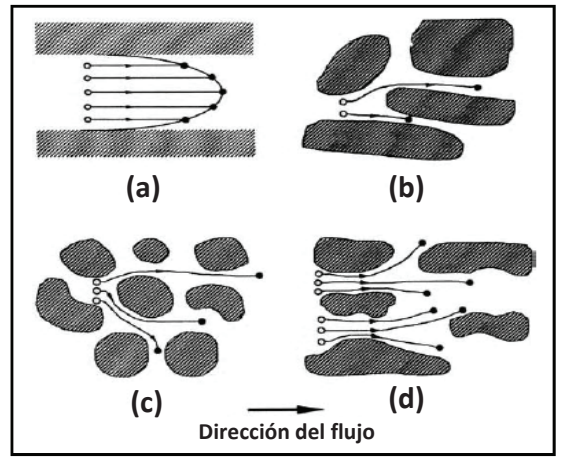

Figura 4. Procesos que alteran el flujo homogéneo de solutos dentro del suelo: (a) dispersión hidrodinámica dentro del poro por roce en las paredes; (b) dispersión por cambio en el tamaño de los poros; (c) procesos de difusión; (d) otros procesos (precipitación, adsorción). Adaptado de Leij y van Genuchten (2002).

Figure 4. Processes that alter the homogeneous flux of solutes through the soil: (a) hydrodynamic dispersion within the pore by friction against the walls; (b) dispersion due to changes in porosity size; (c) diffusion processes; (d) other processes (precipitation, adsorption). Adapted from Leij and van Genuchten (2002). de agua (en unidades de longitud ${ }^{3}$ longitud $^{-3}$ ) y dc/dx son los cambios de concentración en coordenadas espaciales. En condiciones saturadas, $\mathrm{D}_{\mathrm{h}}$ está relacionado linealmente a las tasas de flujo, por lo que se asume un coeficiente de dispersividad $\lambda$ (donde $\mathrm{Dh}=\lambda^{\mathrm{n}}$, siendo $\mathrm{n}$ una constante empírica), el cual depende de las propiedades de suelo, con valores que varían entre 0,01 y $10 \mathrm{~cm}$ en experimentos de pequeña escala, a más de $100 \mathrm{~cm}$ en estudios a escala regional.

Otro fenómeno típico durante el movimiento de solutos en el suelo es la difusión $\left(J_{d}\right)$, la cual denota la migración relativa de un soluto en relación al solvente, el cual puede estar o no en movimiento (Bohne, 2005). La difusión tiende a igualar concentraciones dentro de una solución, estando determinada en los suelos por:

$$
J_{d}=-\theta D_{\text {dif }} \cdot d c / d x
$$

Donde $\mathrm{D}_{\text {dif }}$ corresponde al coeficiente de difusión molecular o iónica para la fase líquida en el suelo y considera parámetros de tortuosidad del medio poroso (Leij y van Genuchten, 2002). $D_{\text {dif }}$ puede ser calculado por la ecuación de Einstein-Stokes (Hillel, 1998), y en general se presenta en rangos desde menos de $0,1 \mathrm{~cm}^{2} \mathrm{~d}^{-1}$ en arcillas compactadas a $1,7 \mathrm{~cm}^{2} \mathrm{~d}^{-1}$ en sedimentos sueltos con alto contenido de agua (Leij y van Genuch-ten, 2002).

En definitiva, el flujo de un soluto a través de la fase líquida del suelo ocurre por convección, dispersión hidrodinámica y difusión, aunque las últimas dos no son fáciles de distinguir, por lo que muchos autores hablan del coeficiente de difusión/dispersión efectivo (Radcliffe y Rasmussen, 2002). Sin embargo, hay que considerar reacciones de naturaleza química, las cuales pueden ser clasificadas según su velocidad y el número de etapas o fases para lograr un cambio. Una de las más comunes corresponde a la adsorción de solutos en coloides orgánicos e inorgánicos, pero como no es objetivo de este artículo ahondar en estos aspectos, se recomienda el enfoque de procesos múltiples descritos por Jury y Horton (2004) y los mecanismos de reacción sólido-solución-solutos descritos por Or y Wraith (2002).

\section{Otros factores que afectan el movimiento de contaminantes}

Al ser el suelo un material anisotrópico, una de las mayores dificultades en su caracterización está dada por la variabilidad natural de sus propiedades (Kutílek y Nielsen, 1994). Las características más comunes en la pedogénesis son la estratificación, la horizonación y los cambios texturales dentro del perfil, los que generan cambios en los sistemas porosos que afectan el flujo de agua y el transporte de solutos (Jury et al., 1991). Sin embargo, existen otros procesos que generan variabilidad espacial, entre los que se incluyen los cambios estructurales, la labranza, la hidrofobicidad o repelencia 
al agua y los fenómenos de estratificación por aporte de enmiendas orgánicas (Figura 5), que muchas veces pueden ser inducidos por manejo antrópico. En sí misma la estructuración de los suelos es un factor de anisotropía, generando tortuosidad a nivel micrométrico (Dörner et al., 2013), de ahí la necesidad de trabajar y entender los procesos de transporte de contaminantes a distinta escala (Majdalani et al., 2015).

El gráfico de la Figura 5 (izquierda) muestra la distribución irregular del agua en un suelo de clase textural franco arcillosa, homogéneo en profundidad. En este caso, la aplicación de estiércol $\left(20 \mathrm{Mg} \mathrm{ha}^{-1}\right)$ entre los 10 y $30 \mathrm{~cm}$ de profundidad generó una alta retención de agua, lo que incrementó la tortuosidad y discontinuidad en el sistema poroso hacia los horizontes subsuperficiales. Este principio se utiliza para realizar sellos en rellenos sanitarios, por ejemplo con adición de dispersantes en materiales arcillosos compactos, aunque no se logra un control $100 \%$ efectivo.

El otro caso señalado en la Figura 5 (derecha), se realizó en un suelo franco arenoso, estratificado, cultivado con maíz, donde se aprecia que la huella de tránsito compacta el suelo, generando un mojamiento heterogéneo, con concentración de flujo y riesgo de contaminación con nitratos; en otros perfiles bajo labranza tradicional, el arado induce una ruptura en la continuidad de los macroporos que restringe los flujos preferenciales y limita el abrupto movimiento de contaminantes en profundidad (Fuentes, 2013). Otra forma de generar flujos preferenciales en el suelo es a través de grietas por contracción, en suelos con alto contenido de arcilla (Pathak et al., 2011) o a través de fenómenos de hidrofobicidad en sistemas de labranza que tienden a generar una estratificación de la materia orgánica (Urbanek et al., 2007).

Un aspecto importante para entender el riesgo de un potencial contaminante es la escala de trabajo, la que orientará la estrategia a seguir en los mecanismos de prevención ó remediación (Figura 6).

Por ejemplo, en una cuenca hidrográfica, el flujo hídrico se separa en las divisorias de agua, curvándose y confluyendo hacia las redes de drenaje natural. En este caso, el modelamiento del flujo de agua y de potenciales contaminantes tiene un mayor grado de incertidumbre, dada la mayor variabilidad espacial, la multiplicidad de factores que afectan el fenómeno y la dificultad en la obtención de la información (Nielsen y Wendroth, 2003).

Al analizar el flujo potencial de contaminantes a escala agronómica (detalle de la Figura 6, arriba a la derecha), existe la tendencia a analizar los procesos en forma unidimensional, con flujos descendentes dados por el aporte de lluvia y riego o ascendentes desde las napas subterráneas hacia la superficie. Sin embargo, en una aproximación a una escala más detallada (Figura 6, abajo a la derecha), es bien sabido que los flujos serán multidireccionales, y dependerán no sólo de la anisotropía del sistema poroso, sino de procesos como la absorción de agua por las plantas (que podrían consumir contaminantes en un proceso de fitoremediación) y de procesos de adsorción, complejación, quelación y precipitación de contaminantes, entre otros. En este contexto Dörner y Dec (2008) estudiaron el efecto de la presencia de un pie de arado sobre la generación de flujos preferenciales a lo largo de una catena de suelo. A través de mode-

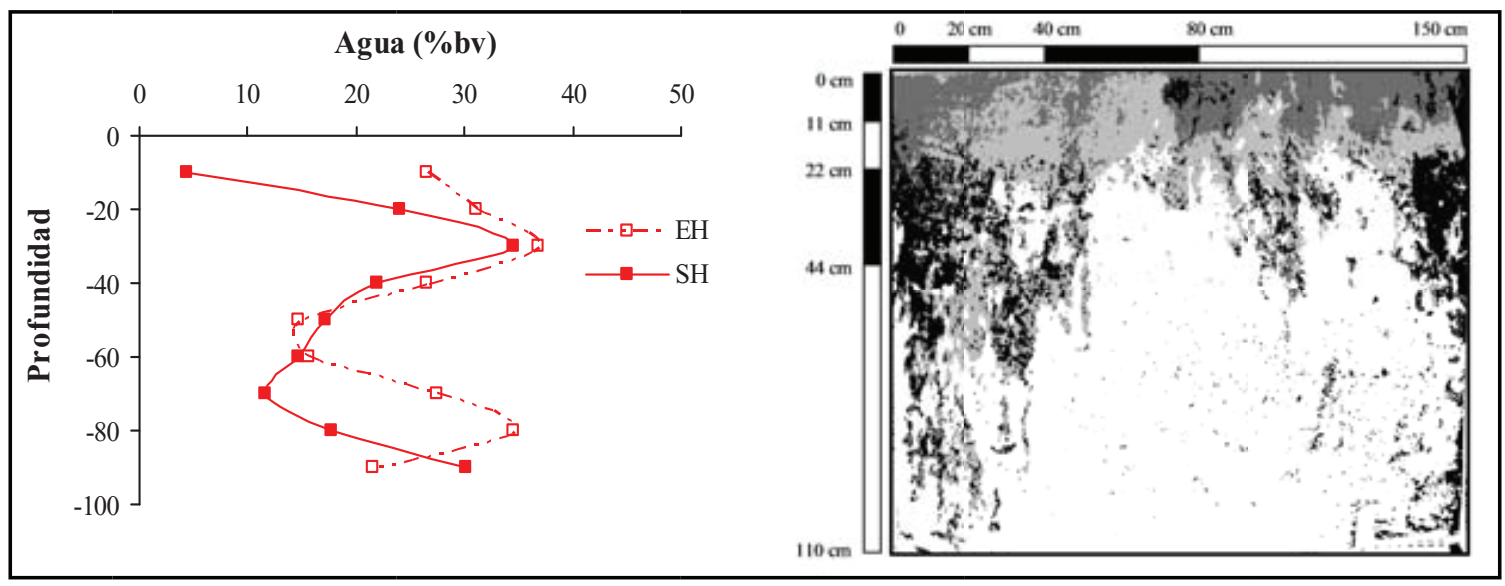

Figura 5. Fenómenos de variabilidad espacial del suelo. A la izquierda, distribución irregular de agua en el perfil (EH: entre hilera, SH: sobre hilera) posterior a un riego en un suelo acondicionado con materia orgánica (adaptado de Keller, 2012); a la derecha, perfil de mojamiento con escala de grises para una tinción de azul brillante (color más oscuro: mayor concentración), donde se observan zonas de flujo preferencial (Fuentes, 2013).

Figure 5. Phenomena of soil spatial variability. On the left, irregular distribution of water into the profile (EH: inter row, SH: on the row) after irrigation in a soil amended with organic matter (adapted from Keller, 2012); on the right, wetting profile in grey scale after staining with blue dye tracer (darker color: higher concentration), with preferential path flows (Fuentes, 2013). 


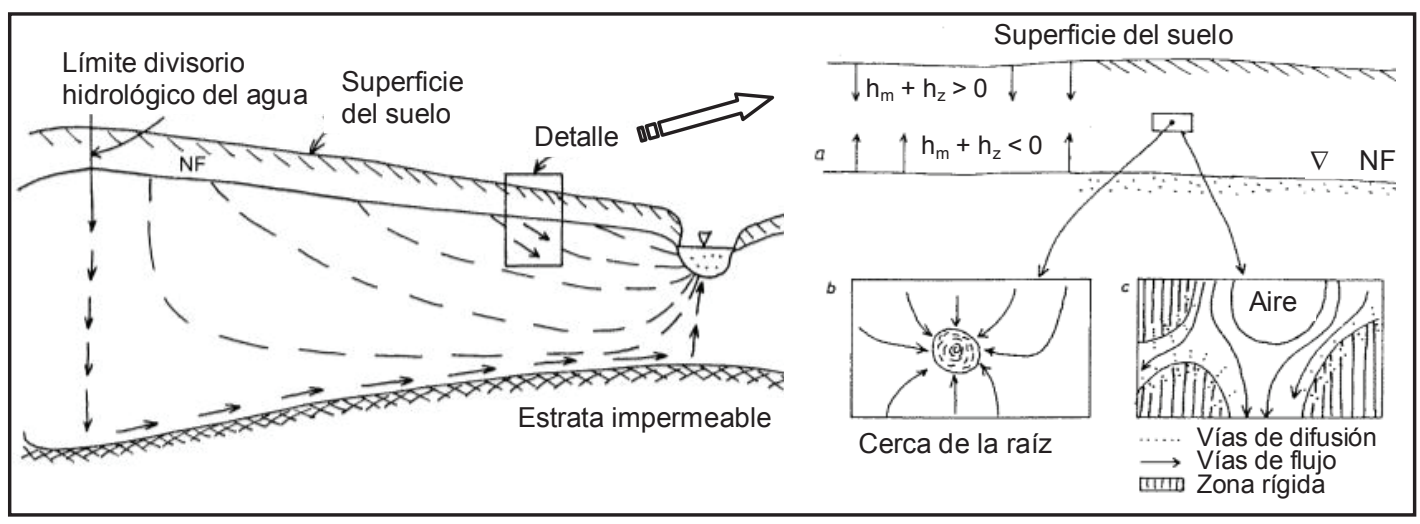

Figura 6. Campos de flujo de agua a distintas escalas. Adaptado de Hartge y Horn (2009).

Figure 6. Water flow vectors at different scales. Adapted from Hartge and Horn (2009).

lación del flujo de agua, demostraron que la anisotropía de la conductividad hidráulica saturada (Ks) desvía el flujo hacia sectores con una mayor Ks; así, dado que un pie de arado genera una estructura laminar, se generó un flujo preferencial paralelo a la pendiente.

La movilidad potencial de contaminantes no se afecta sólo por la variabilidad espacial, también es necesario tener presente el dinamismo temporal del suelo. Por ejemplo, el sistema poroso está continuamente cambiando sus contenidos de agua, entre eventos de riego o a nivel estacional con las precipitaciones, lo que afecta los parámetros de modelamiento (Cuadro 1).

A medida que aumenta el contenido de agua del suelo, aumenta el área de flujo y por ende el coeficiente de difusión/dispersión efectivo (De). Considerando la tortuosidad como L/Lc, en que L es la distancia lineal de flujo y Lc la distancia real de recorrido dentro del sistema poroso (Bohne, 2005), el factor $\xi$ toma en cuenta el aumento de la longitud de recorrido y la disminución del área de sección transversal a medida que el suelo se seca, ponderando con un menor valor la Ecuación (3),

Cuadro 1. Valores de contenido de agua del suelo $(\theta)$ y su

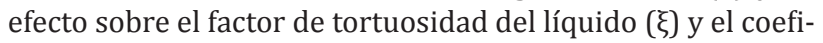
ciente de dispersión efectivo (De).

Table 1. Values of soil water content $(\theta)$ and its effect on liquid tortuosity factor $(\xi)$ and effective dispersion coefficient (De).

\begin{tabular}{ccc}
\hline$\theta\left(\mathbf{c m}^{3} \mathbf{c m}^{-3}\right)$ & $\xi$ & De $\left(\mathbf{c m}^{2} \mathbf{d}^{-1}\right)$ \\
\hline 0,25 & 0,039 & 0,84 \\
0,30 & 0,072 & 3,37 \\
0,35 & 0,120 & 5,82 \\
0,40 & 0,189 & 12,69 \\
\hline
\end{tabular}

ya que las partículas sólidas y el aire de los poros actúan como una barrera a la difusión (Jury et al., 1991).

Para el caso de difusión gaseosa, el análisis es exactamente al revés, ya que el aumento del contenido de agua dificulta el movimiento de gases al interrumpir las vías de flujo (Thorbjørn et al., 2008). En último término, el riesgo de contaminación gaseosa se explica por las mismas variables que en el caso de contaminantes asociados al flujo de agua, principalmente textura y estructura, que resultan en factores de conectividad y tortuosidad (Kunkoro et al., 2014), siendo relevante el rol de la materia orgánica en los factores de estructuración (Hamamoto et al., 2012).

Finalmente, la naturaleza del material que potencialmente se puede movilizar afectará el riesgo de contaminación en función de su solubilidad, volatilidad, capacidad de reacción con otras sustancias del suelo y facilidad de transformación por metabolismo biológico. Así por ejemplo, en un amplio rango de contenido de agua del suelo, existirán pesticidas como el DDT, cuyo coeficiente de difusión es dominado por la fase de vapor, en tanto la atrazina posee una mayor movilidad en fase líquida (Jury y Horton, 2004). Incluso para un mismo elemento, como lo es el caso del C o el N, existen riesgos inherentes debido a la amplia variabilidad de las formas químicas que pueden adoptar. Por ejemplo, en condiciones bien aireadas existirán pérdidas de $\mathrm{CO}_{2}$, en tanto en condiciones de anegamiento el flujo gaseoso será bajo la forma de $\mathrm{CH}_{4}$; para el caso del $\mathrm{N}$, las condiciones de anegamiento favorecen la lixiviación de $\mathrm{NO}_{3}$, pero a la vez se generan las condiciones para las pérdidas de $\mathrm{N}_{2} \mathrm{O}$ (Phillips y Beeri, 2008).

\section{Curvas teóricas de penetración de contaminantes en el suelo}

El mecanismo clásico para entender y definir los factores y parámetros que gobiernan el movimiento de 
contaminantes en el suelo es mediante el uso de columnas experimentales, a través de las cuales se hacen pasar soluciones de distinta naturaleza (Figura 7). Según este esquema, una solución conocida A es desplazada por una solución conocida $B$ en un experimento, donde se toman alícuotas para medir la concentración de solutos en distintos tiempos. Detalles de la teoría y principios se encuentran en Skaggs y Leij (2002), en tanto este y otros métodos experimentales se encuentran descritos en Skaggs et al. (2002).

Si no existiesen fenómenos de dispersión hidrodinámica (Figura 4) o mezcla entre las soluciones A y B, esta última desplazaría a la primera en un frente regu-

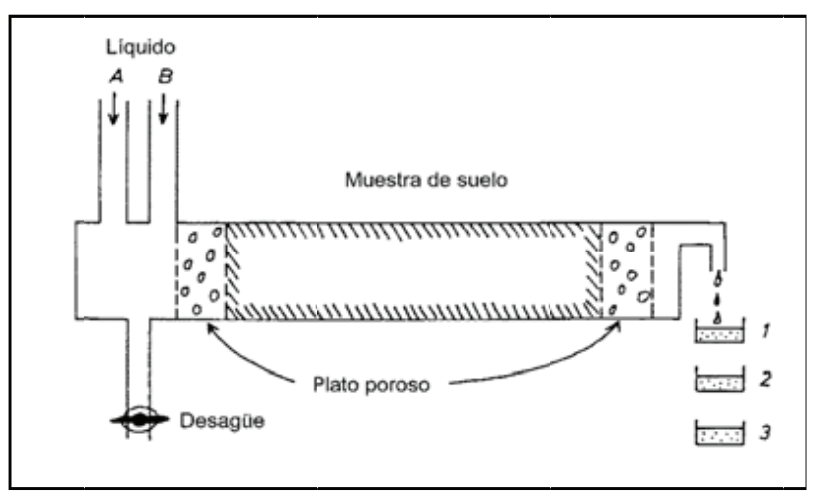

Figura 7. Diagrama esquemático de una columna de suelo usada en laboratorio para experimentos de flujo de contaminantes.

Figure 7. Schematic diagram of a soil column used in laboratory for experiments in pollutants fluxes. lar con forma de pistón (Figura 8, izquierda), pasando en forma instantánea de una concentración relativa (C/Co) igual a cero a una concentración máxima, lo que acusa el cambio repentino en la composición de la solución. Este cambio se produciría en un tiempo o volumen dependiente de la longitud de la columna de suelo.

Sin embargo, debido a las interacciones discutidas en este trabajo, la recta teórica no se cumple, y se comienza a alargar en función de la distancia de recorrido. En forma particular, la aparición de la solución B se puede retrasar en relación a la recta teórica si ocurren fenómenos de sorción o intercambio, mientras que se podría adelantar su aparición si ocurren fenómenos de exclusión de solutos, o incluso podría no llegar a la concentración máxima teórica si ocurre una inmovilización biológica, una transformación o una volatilización (Skaggs y Leij, 2002).

Las curvas de penetración obtenidas permiten sacar ciertas conclusiones respecto a las características del flujo en el medio estudiado. Por ejemplo, el grado de amplitud y asimetría de las curvas es un buen indicador de un desequilibrio físico, mientras que la oblicuidad de las curvas da cuenta del grado de transporte preferencial (Jarvis et al., 2012).

El estudio de desplazamiento de solutos en el suelo se puede realizar a partir de distintas aproximaciones, desde las empíricas en columnas de suelo en laboratorio hasta métodos de campo, con muestreos laboriosos pero que replican de mejor forma las condiciones ambientales y de manejo (Salazar et al., 2011), pasando por diversos modelos específicos o complementarios, por ejemplo, los que modelan procesos hidrológicos (Salazar et al., 2009; Moldrup et al., 2013).

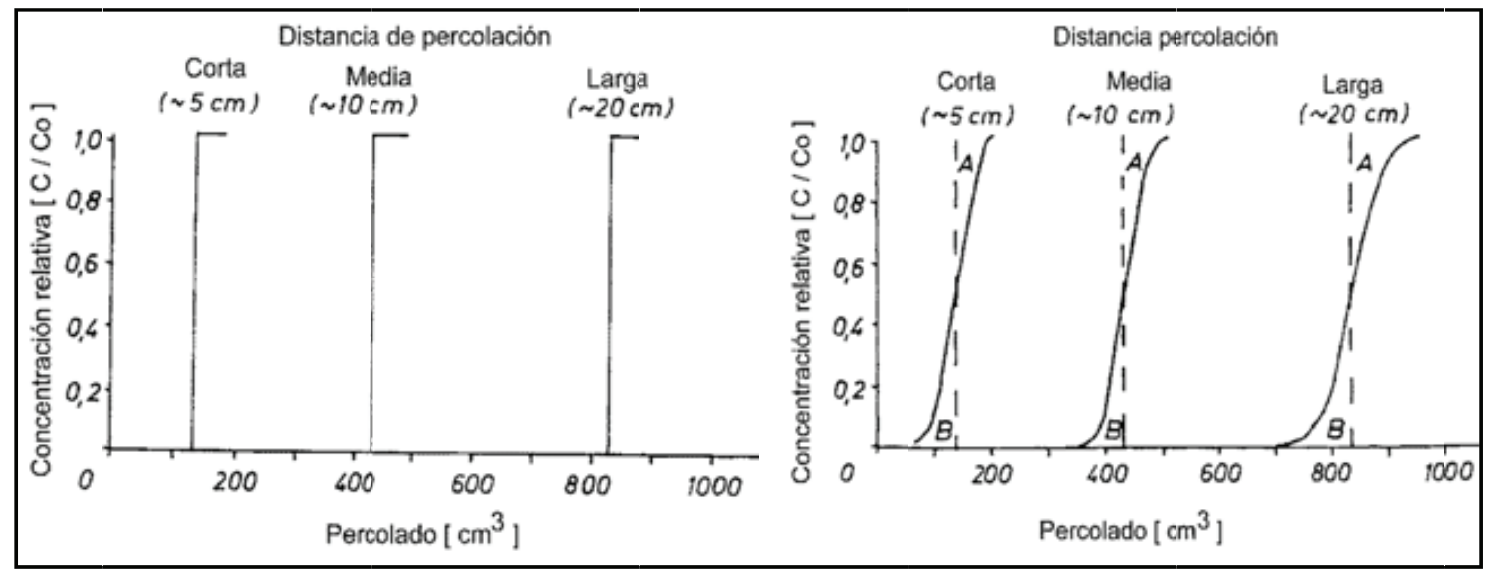

Figura 8. Concentración relativa del flujo de salida de una solución B de concentración conocida. A la izquierda, modelo tipo pistón en que no existe mezcla ni dispersión hidrodinámica; a la derecha, el avance como émbolo se alarga cada vez más respecto a la recta teórica (línea punteada) en función del largo de la columna de suelo. Adaptado de Jury et al. (1991).

Figure 8. Relative concentration of exit flux of a solution B with known concentration. On the left, piston model with no mixing or hydrodynamic dispersion; on the right, the advance as plunger lengthens gradually respect the theoretical line (dotted line) in function of the length of soil column. Adapted from Jury et al. (1991). 


\section{El suelo como filtro ambiental}

Considerando la atmósfera y la hidrósfera como agentes altamente dinámicos, el lugar más seguro para retener y estabilizar contaminantes es el suelo (Bouma, 1996). Su función ambiental puede ser a través de un mecanismo de filtro superficial, en cuyo caso partículas en suspensión dentro de un volumen de agua quedan retenidas en superficie, al infiltrar el agua en el suelo o al evaporarse desde la superficie. En este caso, la efectividad como filtro disminuye en la medida que aumenta la resistencia al flujo en superficie, pero poseen la ventaja de ser reutilizados cuando se eliminan las sustancias filtradas. Sin embargo, lo más común es una función de filtro profundo, en cuyo caso las sustancias disueltas reaccionan con los coloides del suelo, quedando retenidas en este (Stevenson y Cole, 1999). La acumulación y la concentración de una sustancia dependen de la modalidad del filtrado, de allí proviene el término de capacidad de filtro.

Los principios para optimizar el uso del suelo como un filtro ambiental pasan por algunos aspectos básicos, entre los que destacan:

- La capacidad de filtro es mayor mientras más espacio dispone el material que debe ser filtrado; a este respecto, la profundidad de suelo resulta fundamental para asegurar la funcionalidad en el tiempo y la seguridad ambiental.

- Cuando se supera el límite de la capacidad de un elemento, este es transportado desde el sector saturado y retenido en otro a mayor profundidad donde aún existe capacidad de filtro.

- Se requiere una alta proporción de poros, pues los procesos de adsorción necesitan de una gran superficie específica para reaccionar. En ese sentido, un material arcilloso es más efectivo que un material arenoso cuando el contaminante está disuelto en agua.

- El contenido de materia orgánica del suelo también ayuda en el control de los contaminantes, pues aumenta los sitios de intercambio y su capacidad de retención. Sin embargo, es necesario tener presente que, dependiendo del tipo de materia orgánica, el efecto puede variar, ya que los grupos funcionales asociados a ácidos fúlvicos tienden a movilizar ciertos elementos traza metálicos.

- El grado de saturación del filtro debe ser cuidadosamente analizado. Es necesario tener presente que la solución a depurar debe movilizarse dentro del suelo, para lo cual se necesita una buena conductividad hidráulica. Teniendo presente que la conductividad hidráulica saturada es máxima, con un mojamiento de todo el sistema poroso, esta no debe ser alta, de manera de evitar riesgos de percolación profunda, pero tampoco debe ser demasiado baja, de manera de movilizar el contaminante por toda la superficie reactiva del suelo y asegurar una eficiencia en los volúmenes de solución a depurar. Así mismo debe considerarse la estructura, la cual debe ser efectiva en favorecer el flujo de agua, pero no excesiva para evitar la generación de flujos preferenciales.

- La efectividad del filtro aumenta si se complementa con técnicas como la fitoremediación u otras técnicas biológicas, que buscan la inmovilización de los contaminantes en tejidos vegetales que pueden ser fácilmente extraídos del sitio. Un flujo lento significa un mayor tiempo de permanencia y más oportunidades para la descomposición biológica de los compuestos orgánicos.

- Un filtro interno, en contraposición a un filtro superficial, no es absoluto, siempre hay que considerar que en algún sitio pasan sustancias arrastradas por el agua. Al continuar el aporte con estas sustancias, en cualquier momento estas entran en contacto con el agua freática o libre.

\section{CONCLUSIONES}

La movilidad de contaminantes en el suelo puede ser explicado desde el punto de vista de la física de suelos, ya que gran parte de su dinámica depende de los fenómenos de flujo y almacenamiento. Si el agente de interés es soluble, el principal proceso de movimiento dentro del suelo es por flujo de masas; sin embargo, dependiendo de la naturaleza del contaminante y las propiedades del suelo, existirán otros procesos relevantes, como la dispersión hidrodinámica o la volatilización hacia la fase gaseosa.

Resulta de gran importancia conocer las características del suelo que afectan la estructuración, como la textura y el contenido de materia orgánica, que en definitiva determinan el diámetro, continuidad y conectividad del sistema poroso por el cual se mueve un potencial contaminante.

Propiedades como la estructura o la hidrofobicidad, además de las prácticas de manejo, pueden influir en la variabilidad espacial y en la anisotropía del medio poroso, generando zonas de flujo preferencial, los cuales aumentan el riesgo de contaminación.

\section{REFERENCIAS}

Bohne, K., 2005. An Introduction into Applied Soil Hydrology. Lecture Notes in GeoEcology. Catena Verlag, Reiskirchen.

Bouma, J., 1996. Soil protection in relation to soil vulnerability, in: de Haan, F. A. M., Visser-Reyneveld, M. I., (Eds.), Soil Pollution and Soil Protection. International Training Centre, Wageningen, pp. 229-238. 
Campbell, G.S., 1995. Introducción a la biofísica ambiental. EUB. Barcelona, España.

Cuevas, J., Horn, R., Seguel, O., Dörner, J., 2013. Hydraulic conductivity variation in chilean volcanic soils due to wheeling and management. Journal of Soil Science and Plant Nutrition 13(3), 756-766. http://www.scielo.cl/pdf/ jsspn/v13n3/aop6013.pdf

Darcy, H., 1856. Les Fontaines Publiques de la Ville de Dijon. Dalmont, Paris.

Dörner, J., Dec, D., 2008. Efecto de la estructura sobre el movimiento de agua en una catena de suelos. Agro Sur 36(2), 45-52. <http://mingaonline.uach.cl/scielo. php?pid=S0304-88022008000200005\&script=sci_ arttext>

Dörner, J., Dec, D., Peng, X., Horn, R., 2009. Efecto del cambio de uso en la estabilidad de la estructura y la función de los poros de un Andisol (Typic Hapludand) del sur de Chile. Revista de la Ciencia del Suelo y Nutrición Vegetal 9(3), 190-209. http://www.scielo.cl/scielo.php?pid=S071827912009000300003\&script=sci_arttext

Dörner, J., Dec, D., Zúñiga, F., Horn, R., López, I., Leiva, C., Cuevas, J., 2013. Soil changes in the physical quality of an Andosol under different management intensities in southern Chile, in: Krümmelbein, J., Horn, R., Pagliai, M. (Eds.), Soil Degradation. Advances in GeoEcology 42. Reiskirchen. pp. 262-281.

Fuentes, I., 2013. Soil hydraulics associated with soil physical properties and their effect on nitrate leaching in the Mediterranean zone of Chile. Tesis Magíster en Manejo de Suelos y Agua, Universidad de Chile. 124 p.

Hamamoto, S., Moldrup, P., Kawamoto, K., Komatsu, T., 2012. Organic matter fraction dependent model for predicting the gas diffusion coefficient in variably saturated soils. Vadose Zone Journal 11. http://vbn.aau.dk/en/publications/organic-matter-fraction-dependent-model-forpredicting-the-gas-diffusion-coefficient-in-variablysaturated-soils(39e1 ee54-09ad-43d7-bfc1-a22486bcdd0c)/export.html

Hartge, K. H., Horn. R., 2009. Die physikalische Untersuchung von Böden. 4. Aufl. E. Schweizerbart'sche Verlagsbuchhandlung, Stuttgart.

Haygarth, P. M., Jarvis, S. C., 1999. Hydrological factors for phosphorus transfer from agricultural soils. Advances in Agronomy 69, 153-178. http://www.sciencedirect. com/science/article/pii/S0065211308609499

Haygarth, P. M., Condron, L. M., Heathwaite, A. L., Turner, B. L., Harris, G. P., 2005. The phosphorus transfer continuum: Linking source to impact with an interdisciplinary and multi-scaled approach. Science of the Total Environment 344, 5-14. http://www.ncbi.nlm.nih.gov/ pubmed/15907506

Hillel, D., 1980. Fundamentals of soil physics. Academic Press, New York.

Hillel, D., 1998. Environmental soil physics. Academic Press, New York.

Jarvis, N., Messing, I., 1995. Near-saturated hydraulic conductivity in soils of contrasting texture measured by tension infiltrometers. Soil Science Society of American Journal 59, 27-34. <http://dx.doi.org/10.2136/sssaj1995.0361 $5995005900010004 x>$

Jarvbis, N., Moeys, J., Koestel, J., Hollis, J., 2012. Preferential
Flow in a Pedological Perspective, in: Lin, H. (Ed.), Hydropedology. Synergistic Integration of Soil Science and Hydrology. Academic Press, USA, pp. 75-120.

Jury, W. A., Gardner, W. R., Gardner, W. H., 1991. Soil physics. John Wiley \& Sons, Inc. New York.

Jury, W. A., Horton, R., 2004. Soil physics. John Wiley \& Sons, Inc. New Jersey.

Keller, K., 2012. Evaluación de la dinámica del agua en suelos con aplicación de enmiendas orgánicas bajo riego por goteo. Tesis Ingeniero Agrónomo, Universidad de Chile. $42 \mathrm{p}$.

Kunkoro, P. H., Koga, K., Satta, N., Muto, Y., 2014. A study on the effect of compaction on transport properties of soil gas and water. II: Soil pore structure indices. Soil and Tillage Research 143, 180-187. http://dx.doi.org/10.1016/j. still.2014.02.006

Kutílek, M., Nielsen, D. R., 1994. Soil Hydrology. GeoEcology Textbook. Cremlingen.

Leij, F. J., Van Genuchten, M. TH., 2002. Solute transport, in: Warrick, A. W. (Ed.), Soil physics companion. CRC Press, Boca Raton, pp. 189-248.

Maier, M., Schack-Kirchner, H., 2014. Using the gradient method to determine soil gas flux: A review. Agricultural and Forest Meteorology 192-193, 78-95. http://dx.doi. org/10.1016/j.agrformet.2014.03.006

Majdalani, S., Chazarin, J. P., Delenne, C., Guinot, V., 2015. Solute transport in periodical heterogeneous porous media: Importance of observation scale and experimental sampling. Journal of Hydrology 520, 52-60. http://dx.doi. org/10.1016/j.jhydrol.2014.10.065

Moldrup, P., Chamindu Deepagoda, T. K., Hamamoto, S., Komatsu, T., Kawamoto, K., Rolston, D., 2013. Structuredependent water-induced linear reduction model for predicting gas diffusivity and tortuosity in repacked and intact soil. Vadose Zone Journal 12, 1-11. <http://dx.doi. org/10.2136/vzj2013.01.0026>

Nielsen, D. R., Wendroth, O., 2003. Spatial and temporal statistics. Sampling field soils and their vegetation. GeoEcology textbook, Reiskirchen.

Nissen, J., Quiroz, C., Seguel, O., Mac Donald, R., Ellies, A., 2006. Flujo hídrico no saturado en Andisoles. Revista de la Ciencia del Suelo y Nutrición Vegetal 6(1), 9-19. http:// dx.doi.org/10.4067/S0718-27912006000100003

Or, D., Wraith, J. M., 2002. Soil water content and water potential relationships, in: Warrick, A. W. (Ed.), Soil physics companion. CRC Press, Boca Raton, pp. 49-84.

Pathak, P. Wani, S. P., Rao Sudi, R., 2011. Long term effects of management systems on crop yield and soil physical properties of semi-arid tropics of Vertisols. Agricultural Sciences 2(4), 435-442. http://oar.icrisat.org/id/ eprint/4485

Phillips, R., Beeri, O., 2008. The role of hydropedologic vegetation zones in greenhouse gas emissions for agricultural wetland landscapes. Catena 72, 386-394. http:// dx.doi.org/10.1016/j.catena.2007.07.007

Pinochet, D., 2000. El suelo como contaminante: el problema de la contaminación difusa. Boletín № 14: 32-41. En Simposio Proyecto Ley Protección de Suelo. Sociedad Chilena de la Ciencia del Suelo. Comisión Nacional del Medio Ambiente. Valdivia, Chile. 222 p.

Radcliffe, D. E., Rasmussen, T. C., 2002. Soil water movement, 
in: Warrick, A. W. (Ed.), Soil physics companion. CRC Press, Boca Raton, pp. 85-126.

Salazar, O., Wesström, I., Youssef, M. A., Skaggs, R.W., Joel, A., 2009. Evaluation of the DRAINMOD-N II model for predicting nitrogen losses in a loamy sand under cultivation in south-east Sweden. Agricultural Water Management 96(2), 267-281. http://dx.doi.org/10.1016/j. agwat.2008.08.008

Salazar, O., Wesström, I., Joel, A., 2011. Identification of hydrological factors controlling phosphorus concentration in drainage water in sandy soils. Journal of Soil Science and Plant Nutrition 11(2), 31-46. http://dx.doi. org/10.4067/S0718-95162011000200004

Scheffer, F., Schachtschabel, P., 2002. Lehrbuch der Bodenkunde. 15. Aufl. - Spektrum Verlag, Heidelberg.

Skaggs, T. H., Leij, F. J., 2002. Solute transport: Theoretical background, in: Dane, J. H., Topp, G. C. (Eds.), Methods of Soil Analysis. Part 4. Physical Methods. SSSA Book Serie Nr 5. Madison Wisconsin, pp. 1353-1380.

Skaggs, T. H., Wilson, G. V., Shouse, P. J., Leij, F. J., 2002. Solute transport: Experimental Methods, in: Dane, J. H., Topp, G. C. (Eds.), Methods of Soil Analysis. Part 4. Physical Methods. SSSA Book Serie Nr 5. Madison Wisconsin, pp.
1381-1402.

Stepniewska, Z., Fornal, E., Kosiorowska, I., Charytoniuk, P., 2004. Soil aeration and pesticide decomposition, in: Glinski, J., Jozefaciuk, G., Stahr, K. (Eds.), Soil-Plant-Atmosphere aereation and environmental problems. Lublin Suttgart, pp. 94-102.

Stevenson, F. J., Cole, M. A., 1999. Cycles of Soil. Carbon, Nitrogen, Phosphorus, Sulfur, Micronutrients. John Wiley \& Sons, Inc. New York.

Thorbjørn, A., Moldrup, P., Blendstrup, H. Komatsu, T. Rolston, D., 2008. A gas diffusivity model based on air-, solid-, and water-phase resistance in variably saturated soil. Vadose Zone Journal 7, 1276-1286. http://dx.doi. org/10.2136/vzj2008.0023

Trudgill, S. T., Burt, T. P., Heathwaite, A. L., Arkell, B. P., 1991. Soil nitrate sources and nitrate leaching losses, Slapton, South Devon. Soil Use and Management 7, 200-206. http://dx.doi.org/10.1111/j.1475-2743.1991.tb00875.x

Urbanek, E., Hallet, P., Feeney, D., Horn, R., 2007. Water repellency and distribution of hydrophilic and hydrophobic compounds in soil aggregates from different tillage systems. Geoderma 140, 147-155. http://dx.doi. org/10.1016/j.geoderma.2007.04.001 